УДК 519.688

\title{
ОПТИМИЗАЦИЯ ПАРАМЕТРОВ ЭЛЕКТРИЧЕСКОГО ПОЛЯ КАТОДНОЙ ЗАЩИТЫ ОТ КОРРОЗИИ ГАЗОНЕФТЕПРОДУКТОПРОВОДОВ
}

Зенцов Вячеслав Николаевич', zencov.ugntu@yandex.ru

Болотнов Анатолий Миронович², bolotnov@yandex.ru

Удалова Елена Александровна', vakil2004@mail.ru

Мазитова Алия Карамовна', elenaasf@yandex.ru

Лапшакова Ирина Васильевна', irvasya@rambler.ru

Райзер Юлия Сергеевна', julia-2706@mail.ru

Маскова Альбина Рафитовна', asunasf@mail.ru

Васильева Анна Сергеевна', annavas96@mail.ru

1 Уфимский государственный нефтяной технический университет, Россия, 450062, г. Уфа, ул. Космонавтов, 1.

2 Башкирский государственный университет, Россия, 450076, г. Уфа, ул. Заки Валиди, 32.

Актуальность исследования обусловлена встречающимися расхождениями при проектировании катодной защиты трубопроводов. Электрический ток, стекающий с анодного заземления в грунт, распространяется по нему и поступает на защищаемый трубопровод, поляризуя его катод. Для описания закономерностей изменения наложенных потенциалов и силы поляризующего тока по длине трубопровода при катодной защите применяются определенные допущения. При расчете катодной защиты трубопроводов имеются существенные расхождения между проектными и реальными параметрами защиты. В последнее время применяют компьютерные вычислительные эксперименты, позволяющие проводить исследования по проектированию и оптимизации катодной защиты в кратчайшие сроки и меньшими затратами.

Цель. Основываясь на методе фиктивных источников, необходимо предложить алгоритм решения краевой задачи для потенциала электрического поля катодной защиты подземного трубопровода вертикальным глубинным анодом.

объекты: газонефтепроводы, для которых защита от электрохимической коррозии является необходимым условием обеспечения оптимального срока их работы.

Методы: применение метода фиктивных источников обосновано принципом электростатической аналогии для пространственных задач распределения электрического поля. Чтобы применить установленные соотношения к рассматриваемой задаче в полупространстве, в алгоритме дополнительно используется метод зеркальных отражений, позволяющий перевести задачу из полупространства в пространство.

Результаты. Разработана программа на языке программирования $C++$ для проведения численных расчетов электрических полей в системах катодной защиты трубопроводов глубинными анодами с учетом ограничений, накладываемых на потенциал защищаемого сооружения и плотность тока, стекающего с анода. Компьютерная модель дает возможность проведения серий вычислительных экспериментов для выбора геометрических и электрохимических параметров проектируемых глубинных анодов с целью повышения надежности катодной защиты подземных сооружений и увеличения срока их службы. Приведены графики, иллюстрирующие особенности распределений плотности тока вдоль глубинного анода и защитного потенциала трубопровода, при изменениях внешних условий за определенный период эксплуатации. Показано, что при уменьшении сопротивления изоляции для поддержания необходимого защитного потенциала трубопровода требуется увеличение тока катодной станции. Растет плотность тока на границе «анод-грунт», что в свою очередь приводит к более интенсивному растворению анода. Приведены примеры численных результатов, полученных на основе расчетов с реальными исходными данными. Анализ результатов подтверждает эффективность, устойчивость и универсальность разработанного алгоритма.

\section{Ключевые слова:}

Глубинный анод, катодная защита, компьютерное моделирование, метод фиктивных источников,

потенциал, электрическое поле, электрический ток. 


\section{Введение}

В настоящее время система катодной защиты рассчитывается исходя из срока ее службы, равного 15-20 годам. Наибольший эффект катодная защита (К3) трубопроводов дает в том случае, когда трубопроводы имеют хорошую антикоррозийную изоляцию и применяемые анодные заземлители изготовлены из новых композиционных материалов [1].

Промышленностью освоен выпуск станций катодной защиты мощностью от 300 до 5000 Вт со значениями токов $I=12-100$ А. При повышении тока увеличиваются его анодная и катодная плотности, что приводит к более интенсивным процессам газовыделения и электроосмоса, а также к смещению потенциала. Увеличение мощности катодных станций сверх 1000 Вт приводит к увеличению потенциала в точке дренажа выше максимально допустимого [2]. Важным параметром в системе катодной защиты является определение защитной плотности тока, по которой можно рассчитать ток катодных установок. Защитная плотность тока при электрохимической защите газонефтепроводов зависит от состояния изоляционного покрытия и других факторов. Проектные организации для определения тока катодной защиты применяют опытные защиты или проводят физическое моделирование [3-6].

Известно, что электрическое поле в системе «анодный заземлитель - грунт - трубопровод» создается протоком электрического тока через цепь «катодная станция - анодный заземлитель» [7].

Авторы на протяжении последних 30 лет занимаются разработкой новых конструкций анодных заземлителей оптимальных размеров. Организовано промышленное производство анодных заземлителей в коксопековой оболочке [8].

Пространственное распределение электрического поля КЗ определяется многими факторами: режимом работы катодной станции, свойствами грунта, а также геометрическими и электрохимическими параметрами защищаемых сооружений и применяемых анодных заземлителей, значения которых изменяются в процессе эксплуатации [9-11].

Определение и оптимизация параметров КЗ с целью повышения надежности защиты и увеличения срока службы защищаемых сооружений требуют как экспериментальных исследований, так и разработки математических моделей, алгоритмов и комплексов программ для проведения компьютерных экспериментов $[12,13]$.

Основные сложности алгоритмизации задач токораспределения в системах КЗ подземных сооружений связаны с неограниченностью области интегрирования и с большим разбросом значений геометрических параметров объектов. Например, труба диаметром 1 м может иметь участок, защищаемый одним анодом, длиной в десятки километров; вертикальная анодная «гирлянда» с внешним диаметром 10-30 см имеет длину до нескольких десятков метров [14-16].
Компьютерные вычислительные эксперименты, по сравнению с натурными и лабораторными, позволяют проводить исследования по проектированию и оптимизации КЗ с существенно меньшими затратами и в более короткие сроки.

В данной работе описан алгоритм и представлены результаты численных расчетов электрического поля КЗ участка трубопровода глубинным анодом с учетом ограничений, накладываемых на плотность анодного тока и защитный потенциал трубы [17-19].

\section{Математическая модель электрического поля}

Сформулируем краевую задачу для функции потенциала $u(p)$ электрического поля $К 3$ в области $\Omega=\left\{p \mid p=(x, y, z), x \in\left[0, L_{t}\right], y \in(-\infty, \infty), z \in[0, \infty)\right\}$ (рис. 1$)$.
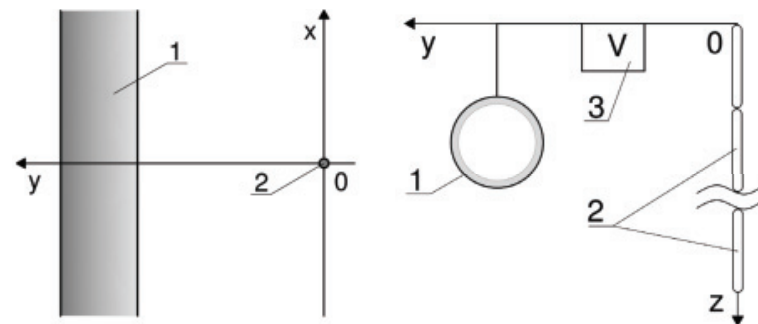

Pис.1. Схема КЗ трубопровода: 1 - труба; 2 - «гирлянда» глубинных анодов; 3 -катодная станиия

Fig. 1. Diagram of short-circuit pipe: 1 is the pipe; 2 is the "garland" of deep anodes; 3 is the cathode station

Известно [20, 21], что потенциал постоянного электрического поля удовлетворяет уравнению эллиптического типа

$$
\operatorname{div}(\sigma(p) \operatorname{grad} u(p))=0, p \in \Omega,
$$

где $\sigma(p)$ - удельная электропроводность среды, $(0 \mathrm{M} \cdot \mathrm{M})^{-1} ; p=(x, y, z)-$ произвольная точка области интегрирования $\Omega$.

Границам-изоляторам $\left(S_{i s}\right)$, к которым отнесем поверхность земли, плоскости сечений трубы (при $x=L_{t}$ ) и анода (при $z=L_{a}$ ) соответствуют частные производные по нормали второго рода

$$
\left.\frac{\delta u}{\delta n}\right|_{S_{i s}}=0,
$$

где $n$ - вектор нормали к границе.

На границах «анод-грунт» $\left(S_{a}\right)$ и «грунт-труба» $\left(S_{t}\right)$ ставятся частные производные по нормали третьего рода:

$$
\left.\left(u+c_{a} \sigma \frac{\delta u}{\delta n}\right)\right|_{s_{a}}=\left.u_{a}\left(u-c_{t} \sigma \frac{\delta u}{\delta n}\right)\right|_{s_{t}}=u_{t},
$$

где $u$ - потенциал в приграничном грунте; $c_{a}$ и $c_{t}-$ удельные сопротивления оболочки анода и изоляции трубы, Ом² $; u_{a}, u_{t}$ - потенциалы сердечника анода и металла трубы; здесь и далее индекс «a» относится к аноду, «t»- к трубе.

Перед вторым слагаемым для анода стоит знак «十», для трубы «-», т. к. направление тока принято положительным от анода в грунт и от грунта к трубе. 
Если зависимости плотности тока от разности потенциалов на границах $S_{a}$ и $S_{t}$ линейны, то параметры $c_{a}$ и $c_{t}$ постоянны для конкретного варианта расчета. Параметр $c_{a}$ может зависеть от координат точки на поверхности анода при моделировании составных анодов переменного радиуса или при учете процесса растворения оболочки анода. Параметр $c_{t}$ может зависеть от положения точки на поверхности трубы при наличии неоднородностей изоляции, что является необходимым в задаче моделирования дефектов в изоляции трубопровода [20-24].

Учитывая, что длины анода и трубы значительно превышают их диаметры, потенциалы металлов естественно считать постоянными в нормальных сечениях, т. е. зависящими только от продольной координаты: $u_{a m}=u_{a m}(z), u_{t m}=u_{t m}(x)$.

В сечениях анода $(z=0)$ и трубы $(x=0)$ ставятся условия второго рода

$$
\left.\frac{d u_{a m}}{d z}\right|_{z=0}=\frac{I_{0}}{\sigma_{a} S_{a m}} ;\left.\frac{d u_{t m}}{d x}\right|_{\tilde{o}=0}=\frac{I_{0}}{2 \sigma_{t} S_{t m}},
$$

где $\sigma_{a}, \sigma_{t}$ - удельные электропроводности металлов анода и трубы; $S_{a m}, S_{t m}$ - площади их «металлических» сечений; $I_{0}$ - ток катодной станции, А.

Так как защищаемый участок трубы симметричен относительно плоскости Y0Z, расчет проводится только для одной половины, поэтому во второй формуле присутствует коэффициент «2» в знаменателе.

Дополнительным условием

$$
\left.\left(u-u_{t m}\right)\right|_{x=L_{t}}=u_{\text {protect }}
$$

обеспечивается необходимое значение защитного потенциала $\left(u_{\text {protect }}\right)$ трубы в точке, наиболее удаленной от анода.

\section{Алгоритм численного решения}

Для алгоритмизации пространственной задачи (1)-(5) применяется метод дискретизации, основы которого заложены в работах В.Н. Ткаченко [25]. Для моделирования электрического поля КЗ магистральных трубопроводов гибкими протяженными анодами аналогичный подход использовался в работе [26], где он назван методом фиктивных источников. Последнего названия мы придерживаемся в данной публикации.

Для перехода от непрерывной модели к дискретной представим глубинный анод в виде $N$ конечных объемных элементов (КОЭ) длиной $\frac{L_{a}}{N}$, а защищаемый участок трубопровода условно разобьем на $M$ элементов длины $\frac{L_{a}}{\grave{I}}$. Далее для каждого КОЭ будем оперировать средними значениями неизвестных параметров:

- $u_{a m}, u_{t m}$ - потенциал в металле КОЭ анода или трубы;

- $u_{a g}, u_{t g}-$ потенциал в грунте, граничащем с КОЭ;

- $I_{a z}, I_{t x}-$ продольный ток в металле между соседними КОЭ;
- $I_{a g}, I_{t g}-$ ток, протекающий через боковую поверхность КОЭ.

При построении алгоритма каждый КОЭ анода и трубопровода будем ассоциировать с фиктивным источником (ФИ), расположенным в геометрическом центре элемента.

Применяя 1-й закон Кирхгофа к каждому ФИ, с учетом (2), сформируем первый блок $N+M$ уравнений:

$$
\begin{gathered}
I_{0}-I_{a z, 1}-I_{a g, 1}=0 ; \\
I_{a z, i}-I_{a z, i+1}-I_{a g j+1}=0(i=1, \ldots, N-2), \\
I_{a z, N-1}-I_{a g, N}=0 ; \\
\frac{I_{0}}{2}-I_{t x, 1}-I_{t g, 1}=0 ; \\
I_{t x, j}-I_{t x, i+1}-I_{t g, j+1}=0(j=1, \ldots, M-2), \\
I_{t x, M-1}-I_{t g, M}=0 .
\end{gathered}
$$

В соответствии с условиями (3) сформируем второй блок $N+M$ уравнений:

$$
\begin{aligned}
& u_{a g, j}+c_{a} \frac{I_{a g, j}}{S_{a, i}}=u_{a m i j}, \quad(i=1, \ldots, N) ; \\
& u_{t g, j}-c_{t} \frac{I_{t g, j}}{S_{t, j}}=u_{t m, j}, \quad(j=1, \ldots, M),
\end{aligned}
$$

где $S_{a i}, S_{t j}$ - площади боковых поверхностей КОЭ, ${ }^{2}$.

Третий блок $N+M-2$ уравнений сформируем из условия выполнения закона Ома между соседними ФИ:

$$
\begin{gathered}
u_{a m, i}-u_{a m, i+1}=\rho_{a} I_{a z j}, \quad(i=1, \ldots, N-1) ; \\
U_{t m, j}-U_{t m, j+1}=\rho_{t} I_{t x, j}, \quad(j=1, \ldots, M-1),
\end{gathered}
$$

где $\rho_{a}, \rho_{t}-$ продольные сопротивления сердечника анода и металла трубы между соседними ФИ, Ом.

Последний блок $N+M$ уравнений связывает потенциалы в грунте на границах КОЭ с интенсивностями точечных ФИ:

$$
\begin{gathered}
4 \pi \sigma u_{a g, i}=\sum_{k=1}^{N} \frac{I_{a g, k}}{R\left(p_{i}, p_{k}\right)}-\sum_{m=1}^{M} \frac{I_{t g, m}}{R\left(p_{i}, p_{m}\right)}, \\
(i=1, \ldots, N) ; \\
4 \pi \sigma u_{t g, j}=\sum_{k=1}^{N} \frac{I_{a g, k}}{R\left(p_{j}, p_{k}\right)}-\sum_{m=1}^{M} \frac{I_{t g, m}}{R\left(p_{j}, p_{m}\right)}, \\
(j=1, \ldots, M),
\end{gathered}
$$

где $R$ - расстояние от ФИ до точки, в которой определяется потенциал, м.

Применение формул (9) обосновано принципом электростатической аналогии для пространственных задач распределения электрического поля. Чтобы применить соотношения (9) к рассматриваемой задаче в полупространстве, в алгоритме дополнительно используется метод зеркальных отражений, позволяющий перевести задачу из полупространства в пространство.

Из условия (5) имеем последнее уравнение:

$$
u_{t g, M}-u_{t m, M}=u_{\text {protect }} \text {. }
$$

Таким образом, сформирована система линейных алгебраических уравнений (6)-(10), в которой число уравнений и неизвестных равно $4(N+M)-1$. 


\section{Вычислительный эксперимент}

В соответствии с изложенным выше алгоритмом авторами разработана программа на языке $C++$. Ниже приведены некоторые результаты расчета КЗ трубопровода глубинным анодом; исходные данные представлены в таблице.

таблица. Значения основных параметров

Table. $\quad$ Values of basic parameters

\begin{tabular}{|c|c|c|}
\hline $\begin{array}{l}\text { Параметр } \\
\text { Paramete }\end{array}$ & $\begin{array}{c}\text { Единица } \\
\text { измерения/Unit }\end{array}$ & $\begin{array}{c}\text { Значение } \\
\text { Value }\end{array}$ \\
\hline $\begin{array}{l}\text { Длина защищаемого участка трубы } \\
\text { (половина) } \\
\text { Length of the protected section } \\
\text { of the pipe (half) }\end{array}$ & $\mathrm{KM} / \mathrm{km}$ & 5,2 \\
\hline $\begin{array}{l}\text { Глубина от уровня земли до трубы } \\
\text { Depth from ground level to pipe }\end{array}$ & $\mathrm{M} / \mathrm{m}$ & 1,2 \\
\hline $\begin{array}{l}\text { Внешний диаметр трубы } \\
\text { Outer diameter of the tube }\end{array}$ & $\mathrm{MM} / \mathrm{mm}$ & 500 \\
\hline $\begin{array}{l}\text { Толщина стенки трубы } \\
\text { Wall thickness }\end{array}$ & $\mathrm{MM} / \mathrm{mm}$ & 5 \\
\hline $\begin{array}{l}\text { Удельное сопротивление стали } \\
\text { Specific resistance of steel }\end{array}$ & $\mathrm{OM} \cdot \mathrm{M} / \mathrm{Om} \cdot \mathrm{m}$ & $2,45 \cdot 10^{-7}$ \\
\hline $\begin{array}{l}\text { Минимальный защитный потенциал } \\
\text { трубы } \\
\text { Minimum protective potential of the pipe }\end{array}$ & $\mathrm{B} / \mathrm{V}$ & 0,3 \\
\hline $\begin{array}{l}\text { Расстояние между анодом и трубой } \\
\text { Distance between the anode and the pipe }\end{array}$ & $\mathrm{M} / \mathrm{m}$ & 220 \\
\hline $\begin{array}{l}\text { Удельное сопротивление грунта } \\
\text { Ground resistivity }\end{array}$ & $\mathrm{OM} \cdot \mathrm{M} / \mathrm{Om} \cdot \mathrm{m}$ & 24 \\
\hline $\begin{array}{l}\text { Длина анода } \\
\text { Anode length }\end{array}$ & $\mathrm{M} / \mathrm{m}$ & 28 \\
\hline $\begin{array}{l}\text { Диаметр стального сердечника анода } \\
\text { Diameter of anode steel core }\end{array}$ & $\mathrm{MM} / \mathrm{mm}$ & 32 \\
\hline \begin{tabular}{|l|} 
Внешний диаметр анода \\
Outer diameter of the anode
\end{tabular} & $\mathrm{MM} / \mathrm{mm}$ & 160 \\
\hline $\begin{array}{l}\text { Удельное сопротивление анодного } \\
\text { наполнителя } \\
\text { Anode filler resistivity }\end{array}$ & $\mathrm{OM} \cdot \mathrm{M} / \mathrm{Om} \cdot \mathrm{m}$ & 0,01 \\
\hline
\end{tabular}

Зависимости анодных и катодных токораспределений от продольных координат представлены на рис. 2.
Приведенные графики иллюстрируют особенности распределений плотности тока $\left(0,1-3,5 \mathrm{a} / \mathrm{M}^{2}\right)$ вдоль глубинного анода и защитного потенциала трубопровода $(0,3-0,4$ В) при изменениях внешних условий: температура перекачиваемого продукта и окружающего грунта, наличие грунтовых вод, механические нагрузки и т. д. за период эксплуатации катодной защиты трубопроводных систем от 5 до 25 лет [27-29]. Из рис. 2, в частности, видно, что при уменьшении сопротивления изоляции для поддержания необходимого защитного потенциала трубопровода (в примере - 0,3 В) требуется увеличение тока катодной станции, следствием чего является рост плотности тока на границе «анод-грунт», что в свою очередь приводит к более интенсивному растворению анода с увеличением коэффициента электрохимического растворения с 2,72 до 4,2 кг/Агод [9].

\section{Выводы}

1. Оптимизация параметров электрического поля катодной защиты от коррозии трубопроводных систем позволяет устранить расхождения по значениям тока, сопротивлениям растеканию электрического поля и потенциалам между проектными и реальными значениями.

2. Разработана программа для проведения численных расчетов электрических полей в системах катодной защиты, которая дает возможность проводить вычислительные эксперименты для выбора геометрических и электрохимических параметров анодных заземлителей, позволяющих увеличить срок службы до 25 лет.

3. Компьютерные вычислительные эксперименты позволяют проводить исследования и расчеты по проектированию электрохимзащиты в более короткие сроки и с меньшими затратами.

4. Предложенная математическая модель электрического поля позволяет производить расчеты по определению сопротивления изоляции, защитного потенциала и плотности тока на границе «анод-грунт».
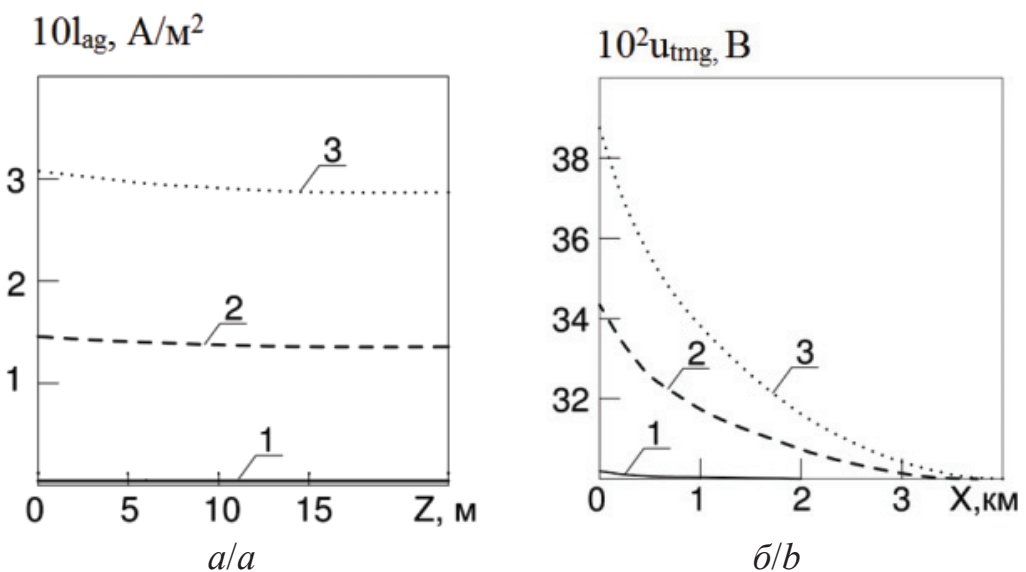

Pис. 2. Продольные распределения плотности тока на гранище «анод-грунт» (а) и защитного потенциала на границе «грунт-тру-

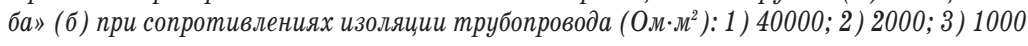

Fig. 2. Longitudinal distribution of current density on «anode-ground» boundary (a) and protective potential on «ground-pipe» boundary (b) at pipeline insulation resistance $\left(\mathrm{Ohm} \cdot \mathrm{m}^{2}\right): 1$ ) 40000; 2) 2000; 3) 1000 


\section{СПИСОК ЛИТЕРАТУРЫ}

1. Зенцов В.Н., Кузнецов М.В. Влияние электроосмоса на работу анодных заземлителей // Транспорт и хранение нефтепродуктов и углеводородного сырья. - 1980. - № 4. - С. 8-9.

2. Optimization of the quantity, locations and output currents of anodes to improve cathodic protection effect of semi-submersible crane vessel / S.H. Xing, Y. Li, H.Q. Song, Y.G. Yan, M.X. Sun // Ocean engineering. - 2016. - V. 113. - P. 144-150. DOI: 10.1016/j.oceaneng.2015.12.047.

3. Современные системы защиты от электрохимической коррозии подземных коммуникаций / Д.Л. Рахманкулов, М.В. Кузнецов, А.И. Габитов, В.Н. Зенцов, А.М. Кузнецов. - Уфа: Издво «Реактив», 1999. - 234 с.

4. Smyrl W.H. Experimental Application of Design Principles in Corrosion Research / Corrosion. - 1979. - V. 35. - № 4. - P. 170.

5. Stress Corrosion Cracking of Carbon Steel in Carbonate Solutions / J.M. Sutcliffe, R.R. Fessler, W.K. Boyd, R.N. Parkins // Corrosion. - 1972. - V. 28. - № 8. - P. 313.

6. Hewes F.W. Four Phenomena Affecting Cathodic Protection and Corrosion Rates // Materials Protection. - 1969. - V. 8. - № 9. P. 67-71.

7. Технология производства заземлителей в коксовой оболочке / М.В. Кузнецов, В.И. Пауль, В.Н. Зенцов, Ф.Г. Тухбатуллин // Современные методы защиты подземных сооружений от коррозии: Материалы семинара. - Л., 1982. - С. 46-50.

8. Глубинный скважинный анодный заземлитель: пат. № 2210628 РФ. № 2002112558/02: заявл. 13.05.2002; опубл. 20.08 .2003 , Бюл. № 2.

9. Лапшакова И.В., Зенцов В.Н. О методах защиты заземленных сооружений от почвенной коррозии // Проблемы строительного комплекса России: Материалы VI Международной научнотехнической конференции. - Уфа: Изд-во УГНТУ, 2002. C. $169-170$.

10. O'Donnel I.P. Lower, Less Variable Power Needs Favor Deep Ground Beds // Oil and Gas Journal. - 1961. - V. 59. - № 8. P. 117.

11. Hatley H.M. Cathodic Protection of pipelines: a deep hole groundbeds // Anti-Corrosion Methods and Materials. - 1968. - V. 15. № 6. - P. 11-12.

12. Проблема аварийности катоднозащищенных трубопроводов / А.М. Кузнецов, В.Н. Зенцов, М.В. Кузнецов, Д.Л. Рахманкулов // Газовая промышленность. - 2001. - № 1. - С. 17-19.

13. Tosato F., Quaia S. Cathodic Protection of Extended Buried Conductor: a New Design Method // IEE Proceedings B - Electric Power Applications. - 1992. - V. 139. - № 5. - P. 443.

14. Deng B., Feng L.J., Yan A.J. Influence of different anodes on cathodic protection for grounding grid // Materials research innovations. - 2013. - V. 17. - P. 126-129. DOI: $10.1179 / 1432891713 Z .000000000202$.

15. Investigation on AC-Induced Corrosion Behavior and Product Film of X70 Steel in Aqueous Environment with Various Ions / H. Zhang, Y. Du, W. Li, M. Lu // Jinshu Xuebao/Acta Metallurgica Sinica. - 2017. - V. 53. - Iss. 8. - P. 975-982. DOI: 10.11900/0412.1961.2016.00566.

16. Bassey 0., Bassey 0.E., Ogedengbe A. Corrosion prevention, control and management: Asset integrity measures in downstream process facilities // New Challenges for a New Era: SPE International Conference and Exhibition on Oilfield Corrosion 2014. Aberdeen, United Kingdom, 2014. - P. 39-62.
17. One-step synthesis of SnCo nanoconfined in hierarchical carbon nanostructures for lithium ion battery anode / J. Qin, D. Liu, X. Zhang, N. Zhao, C. Shi, E.-Z. Liu, F. He, L. Ma, Q. Li, J. Li, C. He // Nanoscale. - 2017. - V. 9. - Iss. 41. - P. 15856-15864. DOI: $10.1039 / \mathrm{c} 7 \mathrm{nr} 04786 \mathrm{j}$.

18. Izadi M., Shahrabi T., Ramezanzadeh B. Active corrosion protection performance of an epoxy coating applied on the mild steel modified with an eco-friendly sol-gel film impregnated with green corrosion inhibitor loaded nanocontainers // Applied Surface Science. - 2018. - V. 440. - P. 491-505. D0I: 10.1016/j.apsusc.2018.01.185.

19. Application of cathodic protection with deep well anode technology to gas-pipeline network in anticorrosive engineering / S.-R. Fu, W.-H. Li, X.-J. Hao, Y.-L. Xu // Corrosion and Protection. - 2013. - V. 34. - Iss. 1. - P. 74-76.

20. Гилязов Т.Т. Методы предотвращения разрушения трубопровода от блуждающих токов // Новые технологии - нефтегазовому региону: Материалы Международной научно-практической конференции студентов, аспирантов и молодых ученых. Тюмень: Тюменский индустриальный университет, 2017. C. $170-172$.

21. Александров 0.Ю., Исупова Е.В., Агиней Р.В. Обзор результатов исследования влияния геомагнитно-индуцированных токов на подземные магистральные нефтегазопроводы // Защита окружающей среды в нефтегазовом комплексе. - 2017. № 1. - С. 44-49.

22. Реализация методов оптимального регулирования режимов работы станций катодной защиты на действующем магистральном газопроводе / С.А. Никулин, Е.А. Спиридович, Р.В. Агиней, Е.Л. Карнавский // Трубопроводный транспорт: теория и практика. - 2015. - № 3 (49). - С. 38-43.

23. Калицев Д.М., Ильин М.С., Кабанец А.А. Анализ методик расчета протекторной защиты магистральных трубопроводов // Фундаментальные и прикладные исследования в современном мире. - 2016. - № 14-1. - С. 162-167.

24. Рахманкулов Д.Л., Зенцов В.Н., Кузнецов М.В. Современная техника и технология защиты от коррозии (теория и практика). - М.: Интер, 2005. - 408 с.

25. Ткаченко В.Н. Анализ поля токов катодной защиты трубопроводной сети // Защита металлов. - 2006. - Т. 42. - № 5. C. $132-135$.

26. Математическая модель и алгоритм расчета электрического поля катодной защиты трубопровода протяженными анодами / А.М. Болотнов, Н.Н. Глазов, Н.П. Глазов, К.Л. Шамшетдинов, В.Д. Киселев // Защита металлов. - 2008. - Т. 44. - № 4. С. $438-441$.

27. Томашов Н.Д. Теория коррозии и защита металлов. - М.: АН СCCP, 1959. - $592 \mathrm{c}$.

28. Pipeline integrity management through internal corrosion monitoring / A.R. Al-Shamari, A.W. Al-Mithin, S. Al-Sulaiman, J. Amer, S. Prakash // NACE - International Corrosion Conference Series. $-2014 .-13 \mathrm{p}$.

29. Janda D., Williams D. Lessons learned monitoring CP current from inside the pipe // Pipeline Pigging and Integrity Management Conference. - Houston, United States, 2014. - 15 p.

Поступила 29.05.2018 2. 


\section{Информация об авторах}

Зенцов В.Н., доктор технических наук, профессор кафедры водоснабжения и водоотведения Уфимского государственного нефтяного технического университета.

Болотнов A.M., доктор физико-математических наук, профессор кафедры высшей математики Башкирского государственного университета.

עдалова $\boldsymbol{E} . \boldsymbol{A}$., доктор технических наук, профессор кафедры физической и органической химии Уфимского государственного нефтяного технического университета.

Мазитова A.K., доктор химических наук, профессор кафедры прикладных и естественнонаучных дисциплин Уфимского государственного нефтяного технического университета.

Лапшакова И.В., кандидат технических наук, доцент кафедры водоснабжения и водоотведения Уфимского государственного нефтяного технического университета.

Paйзер Ю.С., кандидат технических наук, доцент кафедры водоснабжения и водоотведения Уфимского государственного нефтяного технического университета.

Маскова A.P., кандидат технических наук, доцент кафедры прикладных и естественнонаучных дисциплин Уфимского государственного нефтяного технического университета.

Bacuльева A.C., студент кафедры водоснабжения и водоотведения Уфимского государственного нефтяного технического университета. 
UDC 519.688

\title{
OPTIMIZATION OF PARAMETERS OF ELECTRIC FIELD OF GAS PRODUCT AND OIL PIPES CATHODIC PROTECTION
}

Vyacheslav N. Zentsov', zencov.ugntu@yandex.ru

Anatoly M. Bolotnov², bolotnov@yandex.ru

Elena A. Udalova', vakil2004@mail.ru

Aliya K. Mazitova', elenaasf@yandex.ru

Irina V. Lapshakova', irvasya@rambler.ru

Julia S. Rayzer', julia-2706@mail.ru

\author{
Albina R. Maskova', \\ asunasf@mail.ru \\ Anna S. Vasilyeva', \\ annavas96@mail.ru \\ 1 Ufa State Petroleum Technological University, \\ 1, Kosmonavtov street, Ufa, 450062, Russia. \\ 2 Bashkir State University, \\ 32, Zaki Validi street, Ufa, 450076, Russia.
}

The relevance of the research is caused by discrepancies in design of cathodic protection of pipelines. Electric current, flowing from anodic protection to the ground, spreads there and then enters the protected pipeline polarizing its cathode. Certain assumptions are taken to describe the patterns of change imposed by the potential and strength of the polarizing current along the length of the pipeline at a cathodic protection. There are significant differences between design and actual parameters of protection in settlements of cathodic protection of pipelines.

The main aim of the research is to propose an algorithm for solving a boundary value problem for the potential of the electric field of cathodic protection of an underground pipeline by a vertical depth anode based on the method of fictitious sources.

Objects: gas and oil pipelines, for which protection against electrochemical corrosion is a necessary condition for ensuring an optimum period of their operation.

Methods. Based on the method of fictitious sources the authors have proposed the algorithm for solving the boundary-value problem for capacity of the electric field of cathodic protection of underground pipeline vertical deep anode. Application of the method is justified by the principle of electrostatic analogy for spatial task of electric field distribution.

Results. To apply the established relation to this problem in half, the method of mirror reflections is used additionally in the algorithm that allows transferring the task from half to space. The $\mathrm{C}++$ computing code is developed for numerical calculations of electric fields in the systems of cathodic protection of pipelines via deep anodes subject to the restrictions on the electric potential of the protected structure and the density of current, flowing from the anode. The computer model allows conducting a set of numerical experiments for selection of geometric and electrochemical parameters of designed deep anode, to improve the reliability of cathodic protection for underground structures and increase the duration of their service life. The paper introduces the graphs illustrating the features of current density distributions along the depth of anode and the protective capacity of the pipeline, with changes in the external environment for a certain period of operation. It was shown that at decrease in insulation resistance the increase in cathodic station current is required for maintaining the necessary protective pipeline capacity. Current density at the "anode-ground» boundary increases that leads in its turn to more intensive anode dissolution. The paper introduces the examples of numerical results obtained based on real data conditions. The analysis of the results confirms the effectiveness, stability and versatility of the algorithm developed.

Key words:

Deep anode, cathodic protection, computer simulation, method of fictitious sources, potential, electric field, electric current. 


\section{REFERENCES}

1. Zentsov V.N., Kuznetsov M.V. Vliyanie elektroosmosa na rabotu anodnykh zazemliteley [Influence of electroosmosis on anode earths operation]. Transport $i$ khranenie nefteproduktov $i$ uglevodorodnogo syrya, 1980, no. 4, pp. 8-9.

2. Xing S.H., Li Y., Song H.Q., Yan Y.G., Sun M.X. Optimization the quantity, locations and output currents of anodes to improve cathodic protection effect of semi-submersible crane vessel. Ocean engineering, 2016, vol. 113, pp. 144-150. DOI: 10.1016/j.oceaneng.2015.12.047.

3. Rakhmankulov D.L., Kuznetsov M.V., Gabitov A.I., Zentsov V.N., Kuznetsov A.M. Sovremennye sistemy zashchity ot elektrokhimicheskoy korrozii podzemnykh kommunikatsiy [Modern systems of protection against electrochemical corrosion of underground communications]. Ufa, Reaktiv Publ., 1999. 234 p.

4. Smyrl W.H. Experimental Application of Design Principles in Corrosion Research. Corrosion, 1979, vol. 35, no. 4, pp. 170.

5. Sutcliffe J.M., Fessler R.R., Boyd W.K., Parkins R.N. Stress, Corrosion, Cracking of Carbon Steel in Carbonate Solutions. Cor rosion, 1972, vol. 28, no. 8, pp. 313.

6. Hewes F.W. Four Phenomena Affecting Cathodic Protection and Corrosion Rates. Mater. Protect., 1969, vol. 8, no. 9, pp. 67-71.

7. Kuznetsov M.V., Paul V.I., Zentsov V.N., Tukhbatullin F.G. Tekhnologiya proizvodstva zazemliteley $\mathrm{v}$ koksovoy obolochke [Technology of production of earthing switches in the coke shell]. «Souremennye metody zashchity podzemnykh sooruzheniy ot korrozii. Materialy seminara [Modern methods of protecting underground structures from corrosion. Materials of the seminar]. Leningrad, 1982. pp. 46-50.

8. Zentsov V.N., Akulshin M.D., Kuznetsov A.M., Lapshakova I.V. Glubinny skvazhinny anodny zazemlitel [Deep Well Anode Grounding Device]. Patent RF, no. 2210628, 2002.

9. Lapshakova I.V., Zentsov V.N. 0 metodakh zashchity zazemlennykh sooruzheniy ot pochvennoy korrozii [About methods of protection of grounded constructions from ground corrosion]. Problemy stroitelnogo kompleksa Rossii. Materialy VI Mezhdunarodnoy nauchno-tekhnicheskoy konferentsii [Problems of a building complex of Russia. Proc. of the VI International scientific and technical conference]. Ufa, UGNTU Publ. house, 2002. pp. $169-170$.

10. O'Donnel I.P. Lower, Less Variable Power Needs Favor Deep Ground Beds. Oil and Gas Journal, 1961, vol. 59, no. 8, pp. 117.

11. Hatley H.M. Cathodic Protection of pipelines: a deep hole groundbeds. Anti-Corrosion Methods and Materials, 1968, vol. 15, no. 6, pp. 11-12.

12. Kuznetsov A.M., Zentsov V.N., Kuznetsov M.V., Rakhmankulov D.L. Problema avariynosti katodnozashchishchennykh truboprovodov [Problem of failure rate of cathodically protected pipelines]. Gazovaya promyshlennost, 2001, no. 1, pp. 17-19.

13. Tosato F., Quaia S. Cathodic Protection of Extended Buried Conductor: a New Design Method. IEE Proceedings B - Electric Power Applications, 1992, vol. 139, no. 5, pp. 443.

14. Deng B., Feng L.J., Yan A.J. Influence of different anodes on cathodic protection for grounding grid. Materials research innovations, 2013, vol. 17, pp. 126-129. DOI: 10.1179/1432891713Z.000000000202.

15. Zhang H., Du Y., Li W., Lu M. Investigation on AC-Induced Corrosion Behavior and Product Film of X70 Steel in Aqueous Environment with Various Ions. Jinshu Xuebao/Acta Metallurgica Sinica, 2017, vol. 53, Iss. 8, pp. 975-982. D0I: $10.11900 / 0412.1961 .2016 .00566$

16. Bassey 0., Bassey 0.E., Ogedengbe A. Corrosion prevention, control and management: Asset integrity measures in downstream process facilities. SPE International Conference and Exhibition on Oilfield Corrosion 2014. New Challenges for a New Era. Aberdeen, United Kingdom, 2014. pp. 39-62.
17. Qin J., Liu D., Zhang X., Zhao N., Shi C., Liu E.-Z., He F., Ma L., Li Q., Li J., He C. One-step synthesis of SnCo nanoconfined in hierarchical carbon nanostructures for lithium ion battery anode. Nanoscale, 2017, vol. 9, Iss. 41, pp. 15856-15864. DOI: $10.1039 / \mathrm{c} 7 \mathrm{nr} 04786 \mathrm{j}$.

18. Izadi M., Shahrabi T., Ramezanzadeh B. Active corrosion protection performance of an epoxy coating applied on the mild steel modified with an eco-friendly sol-gel film impregnated with green corrosion inhibitor loaded nanocontainers. Applied Surface Science, 2018, vol. 440, pp. 491-505. D0I: $10.1016 /$ j.apsusc.2018.01.185.

19. Fu S.-R., Li W.-H., Hao X.-J., Xu Y.-L. Application of cathodic protection with deep well anode technology to gas-pipeline network in anticorrosive engineering. Corrosion and Protection, 2013, vol. 34, Iss. 1, pp. 74-76.

20. Gilyazov T.T. Metody predotvrashcheniya razrusheniya truboprovoda ot bluzhdayushchikh tokov [Methods for preventing the destruction of the pipeline from stray currents]. Novye tekhnologii - neftegazovomu region. Materialy Mezhdunarodnoy nauchno-prakticheskoy konferentsii studentov, aspirantov $i$ molodykh uchenykh [New technologies for oil and gas region. Proc. of the International Scientific and Practical Conference of Students, Postgraduates and Young Scientists]. Tyumen, Tyumen Industrial University Press, 2017. pp. 170-172.

21. Aleksandrov 0.Yu., Isupova E.V., Aginey R.V. Review of the results of the study of geomagnetically induced currents influence on underground oil and gas pipelines. Zashchita okruzhayushchey sredy $v$ neftegazovom komplekse, 2017, no. 1, pp. 44-49. In Rus.

22. Nikulin S.A., Spiridovich E.A., Aginey R.V., Karnavskiy E.L. Realization of methods for optimal control of operating modes of cathodic protection stations on the existing main gas pipeline. Truboprovodny transport: teoriya i praktika, 2015, no. 3 (49), pp. 38-43. In Rus.

23. Kalitsev D.M., Ilin M.S., Kabanets A.A. Analysis of methods for calculating the tread protection of pipelines. Fundamentalnye $i$ prikladnye issledovaniya $v$ sovremennom mire, 2016, no. 14-1, pp. 162-167. In Rus.

24. Rakhmankulov D.L., Zentsov V.N., Kuznetsov M.V. Sovremennaya tekhnika i tekhnologiya zashchity ot korrozii (teoriya i praktika) [Modern equipment and technology of protection against corrosion (theory and practice)]. Moscow, Inter Publ., 2005. $408 \mathrm{p}$.

25. Tkachenko V.N. Analiz polya tokov katodnoy zashchity truboprovodnoy seti [Analysis of the field of currents of cathodic protection of a pipeline network]. Zashchita metallov, 2006, vol. 42, no. 5 , pp. 132-135.

26. Bolotnov A.M., Glazov N.N., Glazov N.P., Shamshetdinov K.L., Kiselev V.D. Matematicheskay model i algoritm rascheta elektricheskogo polya katodnoy zashchity truboprovoda protyazhennymi anodami [Mathematical model and algorithm for computing the electric field of pipeline cathodic protection with extended anodes]. Protection of Metals, 2008, vol. 44, no. 4, pp. 408-411.

27. Tomashov N.D. Teoriya korrozii $i$ zashchity metallov [Theory of Corrosion and Protection of Metals]. Moscow, AN SSSR Publ., $1959.592 \mathrm{p}$.

28. Al-Shamari A.R., Al-Mithin A.W., Al-Sulaiman S., Amer J., Prakash S. Pipeline integrity management through internal corrosion monitoring. NACE - International Corrosion Conference Series, 2014. pp. 13.

29. Janda D., Williams D. Lessons learned monitoring CP current from inside the pipe. Pipeline Pigging and Integrity Management Conference. Houston, United States, 2014. 15 p.

Received: 29 May 2018. 


\section{Information about the authors}

Vyacheslav N. Zentsov, Dr. Sc., professor, Ufa State Petroleum Technological University.

Anatoly M. Bolotnov, Dr. Sc., professor, Bashkir State University.

Elena A.Udalova, Dr. Sc., professor, Ufa State Petroleum Technological University.

Aliya K. Mazitova, Dr. Sc., professor, Ufa State Petroleum Technological University.

Irina V. Lapshakova, Cand. Sc., associate professor, Ufa State Petroleum Technological University.

Julia S. Rayzer, Cand. Sc., associate professor, Ufa State Petroleum Technological University.

Albina R. Maskova, Cand. Sc., associate professor, Ufa State Petroleum Technological University.

Anna S. Vasilyeva, student, Ufa State Petroleum Technological University. 